\title{
Topological Optimization of Aircraft Frame Structures with the Variable Density Method
}

\author{
Li ying-lei ${ }^{1}$, Cao zong-jie ${ }^{1}$ and Wang Zi-li ${ }^{2}$ \\ ${ }^{1}$ Aviation University of Air Force, School of Aviation Operations and Services, Changchun, 130022, Jilin, China \\ ${ }^{2}$ Aviation University of Air Force, Foreign Training Group, Changchun, 130022, Jilin, China
}

\begin{abstract}
In this paper, a variable density topological optimization method is derived on the basis of Lagrange function, and the RAMP interpolation model is selected to optimize the frame structure of aircrafts with variable density method. For an example, the ordinary frame structure of the domestic planes is taken to illustrate validity of the presented method. The numerical model of the aircraft frames is obtained. The optimal design analysis of the model structural distribution and the weight loss requirement of the aircraft frame structures are realized. In conclusion, the structural distribution law of the topology optimization is summarized according to the topology optimization structures.
\end{abstract}

\section{Introduction}

With the development of aviation, the aircraft with the better comprehensive performances is adapt to the demand of modern wars [1]. Therefore, not only the aerodynamic performance of aircraft structures is necessary [2], the implementation of aviation structural lightweight also has significant practical application value in the structural design [3]. Topology optimization is an optimal method to achieve optimal material layout and lightweight structure when meeting the design constraints [4]. Variable density method, such as the topological optimization methods, is widely used in the topological optimization design of structural continuum [5]. For example, Airbus has applied the topological optimization technique to A380 aircraft on leading edge floor and fuselage door deep floor to achieve a weight reduction of one ton [6]. In the design of the F-35 fighter, the whole structure of the aircrafts was topologically optimized and 25 percent weight reduction of aircraft structures was carried out by Lockheed Martin [7]. In the design of the Boeing 787 aircrafts, a weight reduction of about $25 \%-45 \%$ compared to the corresponding edge floor part of Boeing 777 aircrafts can be achieved with the topological optimization methods [8]

With the topological optimization method based on variable density method, the material distribution of a certain type of domestic aircraft frame structures according to the characteristics about the ordinary frame of the aircrafts is optimally designed in this paper. The best material distribution of the structures is obtained and the weight reduction. to the maximum optimization of the structural materials is realized under the constant loads.

\section{Principle of Variable Density Method}

The variable density method is also called the pseudo density method [9]. It is assumed that there is a functional relationship between the material density of the structure and several physical parameters of the material. The density of the materials can be obtained between the intervals $[0,1]$. The pseudo density about the continuous values arbitrarily in $[0,1]$ of the topological optimization structures is obtained, there are three different situations. If the unit is an empty hole, then the pseudo density of the unit is 0 . If the unit is an entity, its pseudo density unit should be 1 , and if the pseudo density near 0.5 , then introducing the power exponent to penalize this intermediate value, and to make the function value incline to the end value 0 or 1 as much as possible. The pseudo density formulation [10] of the design variables can be expressed as: $\rho=X_{e} \rho_{0}$ where $X_{e}$ is the relative density of each unit; $\rho_{0}$ is the intrinsic density of each unit; $\rho$ is the density of each unit. When $X_{e}$ is equal to 1 , which unit of the materials will be preserved. When $X_{e}$ is equal to 0 , which unit of the materials will be removed. Because $\rho$ is not a continuous function, a penalty factor $\mathrm{p}$ is given in order to let the function called as $K_{e}$ continue. $K_{e}=\left(X_{e}\right)^{p} K_{0}$. Where $K_{0}$ is the element stiffness of original structures; $K_{e}$ is the element stiffness of deformation structures. The structural deformation energy is: $C=F^{T} U=U^{T} K U$. 
where $\mathrm{C}$ represents compliance of structures; $\mathrm{F}$ is the node load vector and $\mathrm{U}$ is the node displacement vector.The mathematical topological optimization condition [18] of aircraft structures can be built as:

$$
\left\{\begin{array}{c}
\text { Find: } X=\left(X_{1}, X_{2}, \cdots X_{n}\right) \\
\text { Minimize: } C=F^{T} U \\
\text { Subject to: } f=\frac{V-V_{1}}{V_{0}} \\
0<X_{\min } \leq X_{e} \leq X_{\max } \\
F=K U
\end{array}\right.
$$

where $V_{0}$ is the initial volume of material; $V$ is the effective volume of material at various design point when the design variable $\rho$ is less than $1 ; V_{1}$ is the material volume when the design variable $\rho$ is equal to $1 ; f$ is a percentage of material used; $X_{\min }$ and $X_{\max }$ are the lower and upper limit of the unit relative density, respectively.

\section{Algorithm topological optimization with variable density method}

Usually Lagrange multiplier method is adopted to derive the mathematical model of the frame structure of a plane[10]. When $X_{\max }=1$ and $X_{\min }=0.01$, Lagrange function in the formula (1) can be changed as:

$$
L=C \lambda_{0}\left(V-f V_{0}-V_{1}\right)+\lambda_{1}^{T}(K U-F)+\sum_{e-1}^{N} \lambda_{2}^{e}\left(X_{\min }-X_{e}\right)+\sum_{e-1}^{N} \lambda_{3}^{e}\left(X_{e}-X_{\max }\right)
$$

where $\lambda$ is Lagrange multiplier. Taken the derivation of objective function:

$$
\frac{\partial L}{\partial X_{e}}=\frac{\partial U^{T}}{\partial X_{e}} K U+U^{T} \frac{\partial K}{\partial X_{e}} U+U^{T} K \frac{\partial U}{\partial X_{e}}+\lambda_{0} V_{e}+\lambda_{1}^{T}\left(\frac{\partial K}{\partial X_{e}} U+K \frac{\partial U}{\partial X_{e}}\right)
$$

The structural equilibrium equation of aircraft structures is:

$$
F=K U
$$

After the derivation of equation(4), we have:

$$
\frac{\partial K}{\partial X_{e}} U+K \frac{\partial U}{\partial X_{e}}=0(e=1,2,3, \cdots, N)
$$

According to equation(4) and (5), the equation can be obtained as follows:

$$
\frac{\partial L}{\partial X_{e}}=U^{T} \frac{\partial K}{\partial X_{e}} U+\lambda_{1}^{T} \frac{\partial K}{\partial X_{e}}+\frac{\partial U}{\partial X_{e}}\left(2 U^{T}+\lambda_{1}^{T} K\right)+\lambda_{0} V_{e}
$$

Since $\lambda_{1}^{T}$ can be an arbitrary value, we can obtain the equation from equation (5).

$$
-P\left(X_{e}\right)^{P-1} U_{e}^{T} K_{0} U_{e}+\lambda_{0} V_{e}=0
$$

Combination of equation (5), multiply $X_{e}^{i}$ is

$$
P U_{e}^{T} K_{0} U_{e} X_{e}^{i-1}=\lambda_{0} V_{e} X_{e}^{i}
$$

since

$$
U_{e}^{T} K U_{e} X_{e}^{i-1}=\theta_{e}
$$

From (8), we can obtain:

$$
X_{e}=\left[\frac{P \theta_{e}}{\lambda_{0} V_{e}}\right]^{\frac{1}{i}}\left(X_{\min }<X_{e}<1\right)
$$

Combination of equation (9) and (10), where $j$ represents the structural units with constant density, $j=1$, $2,3, \ldots, n$, and $k$ represents the structural unit when $X_{e}<1$. The iterative equation of $X_{e}$ is :

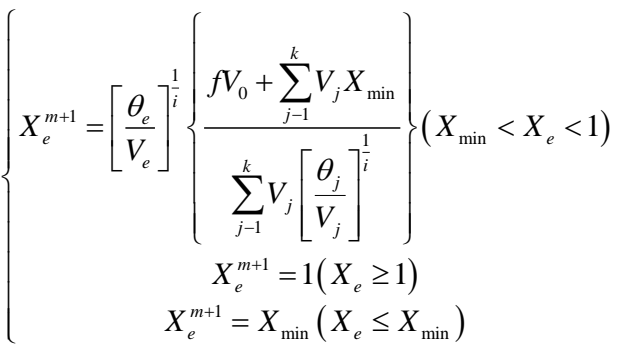

\section{Numerical modelling and analysis of aircraft frame structure}

A certain type of frame structures in domestic aircrafts is selected in this paper, as shown in Figure 1. Specific design parameters are shown in Table 1. Stiffener of the fracture is equal to $5 \mathrm{~mm}$. The frame structure is made of aluminum alloy (type 7075-T7451). Because of its super high strength, the 7075 aluminum alloy is commonly used as aviation manufacturing material, such as highstrength bearing parts like aircraft wing beam, frame and stiffener. According to GB/T/16475-1996, the material properties of the 7075-T7451 aluminum alloy are shown in Table 2. A certain type of three-dimensional geometric aircraft frame is established, as shown in Figure 2. After the completion of the geometric modeling, the workpiece information is transmitted to the preprocessor of the CAE platform in the form of $\mathrm{x} \_\mathrm{t}$ data, as shown in Figure 3 . The physical parameters of the frames include the maximum length, the maximum height and the maximum width. The maximum length of the frames is equal to $1800 \mathrm{~mm}$. The maximum height of the frames is equal to $1800 \mathrm{~mm}$. The maximum width of the frames is equal to $60 \mathrm{~mm}$. The volume of the frames is $6.5357 \times 10^{-3} \mathrm{~m}^{3}$ and the weight of the frames is $51.305 \mathrm{~kg}$.

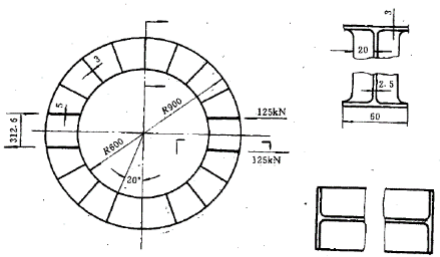

Figure 1. A two-dimensional engineering drawing of an ordinary frame of a certain type of domestic aircraft. 
Table 1. The design parameters of aircraft structures alloy

\begin{tabular}{|c|c|c|c|c|c|}
\hline $\begin{array}{l}\text { Outer } \\
\text { race } \\
\text { radius } \\
(\mathrm{mm})\end{array}$ & $\begin{array}{l}\text { Inner race } \\
\text { radius } \\
(\mathrm{mm})\end{array}$ & $\begin{array}{c}\text { Frame } \\
\text { thick- } \\
\text { ness } \\
(\mathrm{mm})\end{array}$ & $\begin{array}{c}\text { Annulu } \\
\text { s thick- } \\
\text { ness } \\
(\mathrm{mm})\end{array}$ & $\begin{array}{c}\begin{array}{c}\text { Stiff } \\
\text { ener } \\
\text { thick } \\
\text { ness } \\
(\mathrm{mm} \\
)\end{array}\end{array}$ & $\begin{array}{l}\text { Fractur } \\
\text { e width } \\
\text { of the } \\
\text { frame } \\
(\mathrm{mm})\end{array}$ \\
\hline 900 & 60 & 2.5 & 3 & 3 & 312.6 \\
\hline
\end{tabular}

Table 2. The material properties of the 7075-T7451 aluminium

\begin{tabular}{cccccc}
\hline $\begin{array}{c}\text { Parameter } \\
\text { type }\end{array}$ & $\begin{array}{c}\text { Modulus } \\
\text { of } \\
\text { elasticity } \\
(\mathrm{MPa})\end{array}$ & $\begin{array}{c}\text { Poisso } \\
\mathrm{n} \text { ratio }\end{array}$ & $\begin{array}{c}\text { Streng } \\
\text { th } \\
\text { limit } \\
(\mathrm{MPa})\end{array}$ & $\begin{array}{c}\text { Yield } \\
\text { strengt } \\
\mathrm{h}(\mathrm{MPa})\end{array}$ & $\begin{array}{c}\text { Density } \\
\left(\mathrm{g} / \mathrm{mm}^{3}\right)\end{array}$ \\
\hline $7075-$ & $7.2 \times 10^{4}$ & 0.33 & 510 & 470 & $\begin{array}{c}2.83 \times 10^{-} \\
3\end{array}$ \\
\hline
\end{tabular}

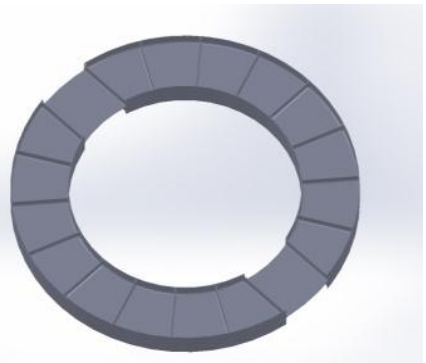

Figure 2. A three-dimensional geometric modeling of an ordinary frame of a certain type of domestic aircraft.

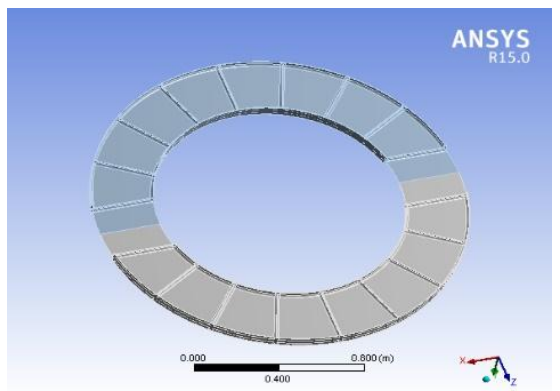

Figure 3. A three-dimensional model of an ordinary frame of a certain type of domestic aircraft.

The partition method selection of frame structure is based on the automatic partition method of entity model and the correlation of grid is set to $0 \sim 80$. Based on the selected physical field characteristics, correlation controls the grid size and to adjust the global grid. The minimum boundary size of the grid is $1.1 \mathrm{~mm}$. As shown in Figure 4, the correlation of the frame structure grid is 80 , as shown in Figure 5, the correlation of the frame structure grid is 0.

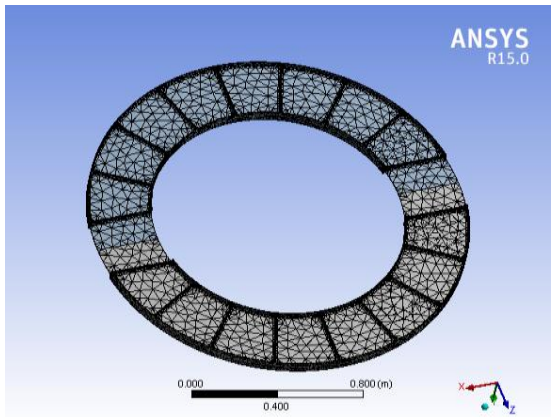

Figure 4. The correlation of the frame structure grid is 80 .

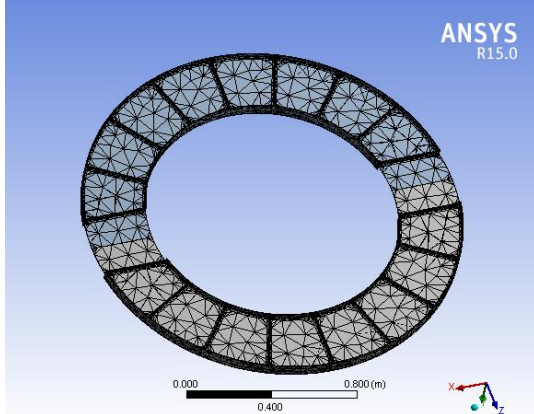

Figure 5. The correlation of the frame structure grid is 0 .

In engineering practice, when the grid correlation is 0 or 80 , the relative error of the workpiece is generally below $3 \%$. The relative error will be reduced with the increase of the grid correlation. This shows that the numerical simulation results have no dependencies on the grid quality because the number of unit and node is more when the grid correlation is greater, then the operation will be more complex, So subsequent analysis in this paper, we take 0 as the correlation of the grid in order to meet the calculation need.

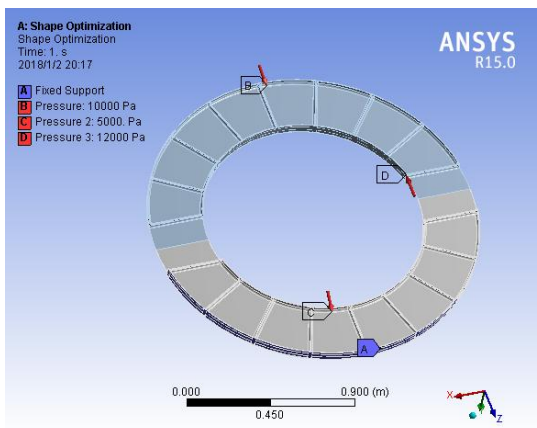

Figure 6. The boundary condition and load of the frame.

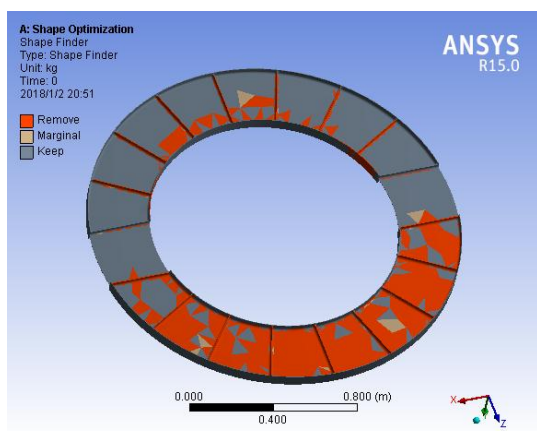

Figure 7. Frame topological optimization nephogram/

The function of the ordinary frame main keeps the shape of the fuselage and provides elastic support to the compressed fuselage skin to prevent them from losing stability. The boundary condition and load of the ordinary frame can be reasonably simplified into the form as shown in Figure 6.

The load distribution will determine the frame optimization space. The percentage of materials is related to the availability of the optimized topological model and the generation of convergence structures. Parameter setting experience of topological optimization in this paper is set the initial value of reducing material 
percentage to $50 \%$. After completing the above steps, optimized iterative calculation is carried out. Actually, the optimization structure with multiple iterations has better static and dynamic performance of the frames. The topological optimization method is automatic multiple iterations in this paper. The topological optimization of an aircraft frame is obtained, as shown in Figure 7.

In Figure 7, the red part is the removable area of the frame, the gray part is reserved area and the brown part is the edge part. When the partial red structural material is removed, the weight of the whole frame can be significantly reduced. The weight of the frame structure is $51.338 \mathrm{~kg}$ before optimization, and it is reduced to $31.726 \mathrm{~kg}$ after optimization, which is reduced by $38.2 \%$. From the analysis results of the frame topological nephogram, it can be concluded that:

(a) The distribution of the material has a great relationship with the load position and load magnitude of the frame. The lower part of the frame structure has a weaker load bearing, so the material is less reserved. And the middle and upper part have a stronger load bearing, so the retention of the material is more concentrated.

(b) The less the percentage of target material is reduced, the less the structure material will be preserved, correspondingly the more difficult the iterative convergence is calculated. But in the design space, most of the unwanted material have been selected certainly in the initial iteration, the subsequent iteration is just used to check the selection process of the boundary material topology.

\section{Conclusion}

The paper mainly makes research on topological technology based on variable density method. By using the three-dimensional modelling of a certain type of domestic aircraft frame, selecting the Lagrange multiplier method as the solving algorithm to derive the variable density method, choosing RAMP as the interpolation model, topological optimization finite element modelling, optimal parameter setting and optimization analysis are all involved in the paper. At last, the reasonable frame structure is optimized, and the material distribution law of the topological optimization is summarized according to the optimized structure analysis.

\section{References}

1 Jin Dongping, Ji bin. Topology optimization of flexible support structure of rear flange of wing $[\mathrm{J}]$. Acta aeronautical journal, 2015,36 (08): 2681-2687.

2 Zhang Ji, Qiu Fusheng. The design of vertical design of aircraft [J]. aircraft based on topology optimization, 2010,30 (03): 31-34.

3 Tong Gang, Liu Tongfei, Deng Yangchen, a wing structure design based on topology optimization. China Science and technology, 2013,10:64-66.

4 Wei Juye. The research of structure topology. 